\title{
The new multidimensional time/multi-frequency transform for higher order spectral analysis
}

\author{
L. Gelman, I. Petrunin
}

Cranfield University, UK

\section{Corresponding author: L. Gelman (1.gelman@cranfield.ac.uk)}

\begin{abstract}
A new multidimensional time/multi-frequency higher order spectral transform is proposed for transient signals with nonlinear polynomial variation of instantaneous frequency: the short time higher order chirp spectra (HOCS) based on the higher order chirp-Fourier transform and time-domain windowing technique. The proposed transform is compared with the classical multi-frequency HOS based on the Fourier transform. It is shown that the proposed transform is more effective for processing of transient processes in comparison with the classical transform.
\end{abstract}

\section{Main notation used}

$x(t)$ is a time domain signal

$x_{v}(t)$ is a time block of signal

$x_{m}(t)$ is a time segment of time block

$h_{e}(t)$ is an external time window

$T$ is a time center of the external window

$h_{i}(t)$ is an internal time window

$H\left(f_{1}, f_{2}, \ldots, f_{n-1}, c_{2}, c_{3}, \ldots, c_{N}, T\right)$ is the HOCS

$n$ is an order of the HOCS

$X_{m}\left(f_{n \Sigma}, c_{2}, c_{3}, \ldots, c_{N}\right)$ is the higher order chirp-Fourier transform

$N$ is an order of the higher order chirp-Fourier transform

$c_{2}$ is the chirp rate (i.e. frequency speed) of the higher order chirp-Fourier transform

$c_{3}$ is the frequency acceleration of the higher order chirp-Fourier transform

$c_{4}, \ldots, c_{N}$ are the higher order parameters of the higher order chirp-Fourier transform

\section{Introduction}

The classical multi-frequency higher order spectra (HOS) based on the Fourier transform has been widely investigated [1-6] for nonlinearity detection in stationary temporal processes. However, for some important applications (e.g. radar and sonar systems, mechanical systems with transient shaft rotation, etc.) it is necessary to process transient temporal processes with any (i.e. linear and nonlinear) polynomial variation of the instantaneous frequency. The classical HOS is not suitable for these processes because

- the stationary kernel of the Fourier transform is not matched with transient processes, including processes with frequency variation in time 
- averaging of HOS for the whole process duration creates estimation errors due to signal nonstationarity

The problem is to create a new HOS based on a transform with kernel matching with any polynomial time variation of the instantaneous frequency. It is important to solve this problem for transient signals from radar, sonar and mechanical systems. This problem has not been investigated in literature.

The higher order chirp-Fourier transform was recently proposed and investigated in ref. [7] for the higher order chirps, i.e. transient signals with any polynomial variation of the instantaneous frequency. Therefore, this transform is suitable for development of a new HOS.

The purposes of this paper are to

- propose a new multidimensional time/multi-frequency higher order spectral technique for transient signals with any (i. e linear and nonlinear) polynomial variation of instantaneous frequency: the short time higher order chirp spectra (HOCS) based on the higher order chirp-Fourier transform and time-domain windowing technique

- compare the proposed HOCS with the classical multi-frequency HOS based on the Fourier transform

The proposed multidimensional HOCS is suitable for the above mentioned signals because

- the non-traditional transient kernel of the higher order chirp-Fourier transform is suitable for transient signals with any polynomial frequency variation

- the time-domain windowing technique is suitable for transient nature of signals

\section{The short time HOCS}

The following steps should be undertaken for estimation of the short time HOCS:

- time domain signal $x(t)$ should be divided into overlapping blocks $x_{v}(t)$ by the external window $h_{e}(t) ; v=1, \ldots, K, K$ defines the total number of blocks $x_{v}(t), T$ is the time center of the external window

- $\quad$ each time block $x_{v}(t)$ should be divided into overlapping segments $x_{m}(t)$ by the internal window $h_{i}(t), m=1, \ldots, M, M$ defines the total number of segments $x_{m}(t)$ in the time block

The general expression of the proposed time/multi-frequency transform, short time HOCS of order $n$ based on the higher order chirp-Fourier transform of order $N$ for each transient block $x_{v}(t)$ can be written as follows:

$$
\begin{aligned}
& H\left(f_{1}, f_{2}, \ldots, f_{n-1,} c_{2}, c_{3}, \ldots, c_{N}, T\right)=\frac{1}{M} \sum_{m=1}^{M} X_{m}\left(f_{1}, c_{2}, c_{3}, \ldots, c_{N}\right) X_{m}\left(f_{2}, c_{2}, c_{3}, \ldots, c_{N}\right) \ldots \\
& X_{m}\left(f_{n-1}, c_{2}, c_{3}, \ldots, c_{N}\right) X_{m}{ }^{*}\left(f_{n \sum}, c_{2}, c_{3}, \ldots, c_{N}\right)
\end{aligned}
$$


where $f_{j}$ is frequency, $j=1, \ldots, n-1, f_{n \Sigma}=\sum_{j=1}^{n-1} f_{j} ; f_{n \Sigma}$ is the accumulated frequency, ${ }^{*}$ is a symbol of a complex conjugate, $X_{m}\left(f_{j}, c_{2}, c_{3}, \ldots, c_{N}\right)$ and $X_{m}\left(f_{n \Sigma}, c_{2}, c_{3}, \ldots, c_{N}\right)$ are the higher order chirp-Fourier transforms [7] at appropriate frequencies for the $m$-th segment $x_{m}(t)$ of the block $x_{v}(t)$,

$$
\begin{aligned}
& X_{m}\left(f_{j}, \quad c_{2}, c_{3}, \ldots, c_{N}\right)=\int_{-\infty}^{\infty} x_{m}(t) e^{-i 2 \pi\left(f_{j} t+\frac{c_{2}}{2} t^{2}+\frac{c_{3}}{3} t^{3}+\ldots+\frac{c_{N}}{N} t^{N}\right)} d t \\
& X_{m}\left(f_{n \Sigma}, \quad c_{2}, c_{3}, \ldots, c_{N}\right)=\int_{-\infty}^{\infty} x_{m}(t) e^{-i 2 \pi\left(f_{n} t+\frac{c_{2}}{2} t^{2}+\frac{c_{3}}{3} t^{3}+\ldots+\frac{c_{N}}{N} t^{N}\right)} d t,
\end{aligned}
$$

$c_{2}$ is the chirp rate (i.e. frequency speed) of the transform, $c_{3}$ is the frequency acceleration of the transform, $c_{4}, \ldots, c_{N}$ are the higher order parameters of the transform; parameters $c_{h}$ characterize the nonlinear polynomial variation of the instantaneous frequency $f_{j i}$ of the transform, $h=2, \ldots, N$.

This variation can be written as follows [7]:

$$
f_{j i}(t)=f_{j}+c_{2} t+c_{3} t^{2}+\ldots+c_{N} t^{N-1}
$$

The time centre of the external window defines the time localization of the external window.

The internal and external windows could be of different types. One can use different external and internal windows: e.g. rectangular, Hamming, Chebyshev, etc. The correlation between external window length and internal window length could be employed from reference [3]. If the signal has fast variations, then a shorter external window $h_{e}(t)$ is required. If, according with reference [3], we maintain the internal window length to be the $(n-1)$ th root of the external window length, then the shorter internal window length is required, which in turn provides poorer frequency resolution. Thus, we have better time resolution and poorer frequency resolution. If it is desired to represent only the long term signal evolution, then it may suffice to use a longer external window. According with reference [3], the longer internal window can be used in this case which in turn provides better frequency resolution. Thus, we have poorer time resolution and better frequency resolution due to the longer internal window

So, it follows from the considered basic cases that selection of the external and the internal window lengths for the short time HOCS is always a trade-off between time and frequency resolutions.

The amount of overlapping provided by the external and internal windows could be also different. Normally, $50 \%$ overlapping is used in the classical HOS for internal windowing in order to increase number of averaged segments and avoid redundancy. It is recommended that the same amount of overlapping be used for shorttime HOCS. However, if signal has fast variations, then a shorter external window $h(t)$ is required; if in this case one wants to maintain the same length of the internal window (i.e. the same frequency resolution) and number $M$ of averaging, then a higher level of overlapping (e.g. 75-80\%) is required. A higher level of overlapping for the internal windowing leads to accuracy increment of HOCS estimation; however, the level of overlapping around $90-95 \%$ is redundant. 
The amount of overlapping provided by the external window depends on the required time resolution. Higher level of overlapping for the external windowing leads to increment of time resolution of HOCS analysis.

The short time HOCS is an $(n+N+1)$ dimensional function, i. e. the real and the imaginary HOCS components, $(n-1)$ frequencies, time and $(N-1)$ parameters $c_{h}$. It is an $n$ dimensional (i.e. time/ $(n-1)$ frequency) transform and generalization of the classical multi-frequency HOS based on the Fourier transform for the case of transient signals with nonlinear polynomial variation of instantaneous frequency.

Physical significance of the proposed technique is that it uses a transform with transient (not stationary) polynomial kernel which is suitable for transient signals with any polynomial variation of the instantaneous frequency in time.

Suppose that the temporal transient signal $x(t)$ is an additive mixture of $(n-1)$ transient components $s_{j}(t)$, narrowband or sinusoidal. In the practically important non-stationary case, instantaneous frequencies of these components vary in time; frequency variations are linear and nonlinear and could be different for different components and different parts of the signal $x(t)$.

Let's consider a generic complex non-stationary component $s_{j}(t)$ with amplitude $A_{j}(t)$ and nonlinear polynomial variation of the instantaneous frequency in time:

$$
s_{j}(t,)=A_{j}(t) e^{j 2 \pi\left[F_{j} t+V_{j}(t)\right]},
$$

where $V_{j}(t)=\left(C_{2 j} t^{2} / 2\right)+\left(C_{3 j} t^{3} / 3\right)+\left(C_{4 j} t^{4} / 4\right)+\ldots+\left(C_{N j} t^{N} / N\right), V_{j}(t)$ is a variation function [7], $F_{j}$ is the initial frequency, $C_{2 j}$ is the constant chirp rate (i.e. the frequency speed), $C_{3 j}$ is the constant frequency acceleration, $C_{4 j}, \ldots, C_{N j}$ are the higher order constant parameters, $t$ is time, $N$ is an order of nonlinear frequency variation, $N \geq 3$.

The nonlinear variation of the instantaneous frequency of the component $s_{j}(t)$ can be written as follows [7]:

$$
F_{j i}(t)=F_{j}+C_{2 j} t+C_{3 j} t^{2}+\ldots+C_{N j} t^{N-1}
$$

Let's assume that frequency variations $F_{j i}(t)$ are known a priori or from independent measurement (e.g. from frequency sensors of radar and sonar systems, tachometer signal of the machinery shaft, etc) for the whole duration of the signal.

If a frequency variation is known a priori, one can use it directly. If a frequency variation is known from independent measurement, one can approximate it firstly by polynomial approximation and then use the obtained approximation for transform adaptation.

In the assumed case, we propose the adaptive signal processing of transient processes by the following twostep adaptation:

- calculate (from the known frequency variation $F_{j i}(t)$ ) for each $m$-th segment and component $s_{j}(t)$ the mean values $\bar{F}_{j i m}$ of the instantaneous frequencies and mean values $\hat{C}_{h j m}$ of the instantaneous 
parameters $C_{h j m}$, i. e. mean values of the chirp rate $C_{2 j m}$, mean values of the frequency acceleration $C_{3 j m}$ and mean values of the higher order parameters $C_{4 j m}, \ldots, C_{N j m}$; parameters $C_{h j m}$ characterize the nonlinear variations $F_{j i m}(t)$ of the instantaneous f1requency during $m$ th segment

- estimate the higher order chirp-Fourier transforms for the $m$ th segment at the instantaneous frequencies $f_{j i m}=\bar{F}_{j i m}$; the chirp rate $c_{2 j m}$, the frequency accelerations $c_{3 j m}$ and the higher order parameters of the transform $c_{4 j m}, \ldots, c_{N j m}$ used for this estimations are equal to the appropriate estimates of the signal: $c_{h j m}=\hat{C}_{h j m}$

Mean values $\bar{F}_{j i m}$ and $\hat{C}_{h j m}$ could be evaluated by the following equations:

$$
\begin{aligned}
& \bar{F}_{j i m}=\frac{1}{\Delta t} \int_{t_{m 1}}^{t_{m 2}} F_{j i}(t) d t, \\
& \hat{C}_{h j m}=\frac{1}{\Delta t} \int_{t_{m 1}}^{t_{m 2}} F^{(h-1)} j i(t) d t,
\end{aligned}
$$

where $\Delta t$ is the segment duration, $t_{m 1}$ and $t_{m 2}$ are time moments of the beginning and the end of the $m-t h$ segment, $F^{(h-1)}{ }_{j i}$ is the $(h-1)$ derivative of the time variation $F_{j i}(t)$.

Let's calculate for each transient block $x_{v}(t)$ the proposed short time HOCS only for $(n-1)$ components $s_{j}(t)$ using this adaptive approach:

$$
H_{s_{j} s_{k} \ldots s_{l}}(T)=\frac{1}{M} \sum_{m=1}^{M} X_{m s_{j}}\left(f_{j i m}\right) X_{m s_{k}}\left(f_{k i m}\right) \ldots X_{m s_{l}}\left(f_{\text {lim }}\right) X_{m s_{j} s_{k} \ldots s_{l}} *\left(f_{i m n \Sigma}\right)
$$

where $f_{V i m}$ is the instantaneous frequency of the signal component $s_{V}(t)$ for the $m t h$ segment; $V=j, k, \ldots, l$, $k=1, n-1, \ldots, l=1, n-1, f_{i m n \Sigma}=\sum_{V=j}^{l} f_{V i m} ; f_{\text {imn } \Sigma}$ is the accumulated instantaneous frequency for the $m t h$ segment, $X_{m s_{V}}\left(f_{V i m}\right)$ and $X_{m s_{j} s_{k} \ldots s_{l}}\left(f_{i m n \Sigma}\right)$ are the higher order chirp-Fourier transforms at the appropriate instantaneous frequencies for the $m$ th segment,

$$
\begin{aligned}
& X_{m s_{V}}=\int_{-\infty}^{\infty} x_{m}(t) e^{-i 2 \pi\left(f_{V m} t+\frac{c_{2 V m}}{2} t^{2}+\frac{c_{3 V m}}{3} t^{3}+\ldots+\frac{c_{N V m}}{N} t^{N}\right)} d t, \\
& X_{m s_{j} s_{k} \ldots s_{l}}=\int_{-\infty}^{\infty} x_{m}(t) e^{-i 2 \pi\left(f_{m n \Sigma} \Sigma^{t+\frac{c_{2 m \Sigma}}{2}} t^{2}+\frac{c_{3 m \Sigma}}{3} t^{3}+\ldots+\frac{c_{N m \Sigma}}{N} t^{N}\right)} d t,
\end{aligned}
$$


$f_{V m}$ the initial frequency of the signal component $s_{V}(t)$ for the $m t h$ segment; $f_{m n \Sigma}=\sum_{V=j}^{l} f_{V m} ; f_{m n \Sigma}$ is the accumulated initial frequency, $c_{h m \Sigma}=\sum_{V=j}^{l} c_{h V m}$.

One can see the differences between the general expression (1) of the short time HOCS and expression (5) of the short time HOCS with the proposed adaptation at each segment. The main difference is that in expression (5) parameters of the HOCS are different for different segments due to adaptation.

One can see that in the considered case the short time HOCS is the three dimensional function (i.e. real and imaginary HOCS components and time). This function can be plotted using the traditional "waterfall" plot. It follows from expression (5) that, using the proposed approach, we cannot specify the particular frequencies at which the short time HOCS is estimated; we can only state that the short time HOCS is estimated for $(n-1)$ components $s_{j}, s_{k}, \ldots, s_{l}$.

The proposed short time HOCS could be also employed for the linear variations of the instantaneous frequencies $F_{j i}$. In this case, equations (1-4) should be used with the following conditions: $c_{3}=\ldots=c_{N}=0$.

\section{Signal modeling}

To compare the proposed and the classical transforms, an input transient random sinusoidal signal with constant amplitude, random initial phase and linearly changed instantaneous frequency in time (i.e. linear chirp) has been passed via the following nonlinear (bilinear) system [8]:

$$
\left\{\begin{array}{l}
\ddot{x}(t)+2 h \dot{x}(t)+\omega_{S}{ }^{2} x(t)=A \cos [\omega(t) t+\varphi], x \geq 0, \\
\ddot{x}(t)+2 h \dot{x}(t)+\omega_{C}{ }^{2} x(t)=A \cos [\omega(t) t+\varphi], x<0
\end{array}\right.
$$

where $x(t)=\frac{X(t)}{m}, X(t)$ is the displacement, $h=\frac{c}{2 m}, \omega_{S}=\sqrt{\frac{k_{S}}{m}}, \omega_{C}=\sqrt{\frac{k_{C}}{m}}, m$ and $c$ are the system mass and damping coefficient respectively, $k_{S}$ and $k_{C}$ are the stiffness for positive displacement (i. e stretching) and stiffness for negative displacement (i. e. compression) respectively, $A=\frac{A_{1}}{m}, A_{1}$ is the constant amplitude of the input signal, $\omega(t)$ and $\varphi$ are the linearly changed circular instantaneous frequency and random initial phase uniformly distributed in the range $[0 ; 2 \pi]$.

The bilinear system (8) is widely used in applications, e.g. for investigation of sub-harmonic resonances of offshore structures: free-hanging risers, tension leg platforms and suspended loads [8-10], articulated loading towers, constrained by a connection to a massive tanker or vessels moored against fenders [11], oscillating parts with clearances and motion limiting stops [12], etc.

System nonlinearity is detected by the proposed short time HOCS of order 3 based on the higher order chirpFourier transform of order 2, (i.e. $n=3, N=2$ ) because the frequency variation of the input signal is linear. 
Parameters used for modelling of the input signal and the system are as follows: the chirp rate of the input signal is $10 \mathrm{~Hz} / \mathrm{s}$; sampling frequency is $20 \mathrm{kHz}$; stiffness ratios $k^{*}=\frac{k_{c}-k_{s}}{k_{c}}$ are 0 (linear system) and 0.1 (nonlinear system); damping $h$ is 250 , the resonance frequencies of the system are $1500 \mathrm{~Hz}$ and $1460.5 \mathrm{~Hz}$ at the stiffness ratios 0 and 0.1 respectively; linear variation of the instantaneous input signal frequency is in the range (1400-1560) Hz.

So, the instantaneous input signal frequency goes through the resonance frequencies of both the linear and the nonlinear systems.

The output signal of the system (8) at resonance consists of the fundamental harmonic of the resonance oscillations (at the stiffness ratio 0, linear system) and of the fundamental and higher harmonics of the resonance oscillations (at the stiffness ratio 0.1, nonlinear system) [10].

Gaussian white noise was added to the output signal to hinder the detection of the system nonlinearity so as to more closely mimic data from the early stage of nonlinearity development; the signal/noise ratio is $40 \mathrm{~dB}$.

Parameters used for estimation of the proposed short time HOCS and classical HOS of the normalized output acceleration $\ddot{x}(t)$ are as follows: segment size is 0 . 4s (i.e. the frequency resolution is $2.5 \mathrm{~Hz}$ ); signal duration is $8 \mathrm{~s}$, overlapping of the internal windows is $50 \%$, the internal window is the Hamming window, the external window is rectangular. The HOCS and HOS were estimated for an external rectangular time-domain window in the vicinity of system resonance.

The higher order transform of order 3 at the fundamental and the second resonance harmonics (i. e. $H_{s_{1} s_{1}}$ ) is normally used for nonlinearity detection for the system (8). In practice, the normalized higher order technique, i. e. skewness [4], is often employed for nonlinearity detection.

The normalized HOCS, skewness based on the higher order chirp-Fourier transform, could be written as follows:

$$
S_{s_{j} s_{k}}(T)=\frac{H_{s_{j} s_{k}}(T)}{\frac{1}{M} \sqrt{\sum_{m=1}^{M}\left|X_{m s_{j}}\right|^{2} \sum_{m=1}^{M}\left|X_{m s_{k}}\right|^{2} \sum_{m=1}^{M}\left|X_{m s_{j} s_{k}}\right|^{2}}}
$$

A total of 800 modeling tests were performed. The values of the detection effectiveness, Fisher criteria [13], for the classical normalized HOS of order 3, skewness, and the normalized HOCS, skewness based on the chirp-Fourier transform (9) at the fundamental and the second resonance harmonics are 240 and 343 respectively. The gain $G$, the ratio of the Fisher criteria for the proposed and the classical techniques, is $G=1.43$

It can be seen that the proposed new time/multi-frequency higher order spectral transform provides a higher value of the detection effectiveness criterion, Fisher criterion, in comparison with the classical multi-frequency transform and is therefore more effective for nonlinearity detection in transient cases.

\section{Conclusions}


1. It is proposed a new multidimensional time/multi-frequency higher order spectral technique for transient signals with known nonlinear and linear polynomial variations of instantaneous frequency: the short time higher order chirp spectra (HOCS) based on the higher order chirp-Fourier transform and time domain windowing technique.

2. The two step adaptation is proposed for the new transform:

- calculate for each segment and signal from the known instantaneous frequency variations the mean values of parameters which characterize these variations

- estimate the HOCS for each segment using parameter values of the transform which are equal to the appropriate mean values of parameters which characterize known variations of instantaneous frequency

For adaptive usage of the proposed transform one needs to know a priori or from independent measurement the instantaneous frequency variation of signal.

3. The bilinear and linear systems under transient cosine excitation with linear variation of the instantaneous frequency were simulated. System nonlinearity was detected by the short time HOCS of order 3 based on the higher order chirp-Fourier transform of order 2 with the proposed adaptation. It was shown that the proposed HOCS is more effective for nonlinearity detection than the classical $\mathrm{H}$ 